declared, M. Glowacka-Kulesz: None declared, I. Szombati: None declared, A. Dudek: None declared, N. Mozaffarian Shareholder of: Gilead, Employee of: Gilead, J. Greer Shareholder of: Gilead, Employee of: Gilead, R. Kunder Shareholder of: Gilead, Employee of: Gilead, D. An Shareholder of: Gilead, Employee of: Gilead, P. Harrison Shareholder of: Galapagos, Employee of: Galapagos, A. Van der Aa Shareholder of: Galapagos, Employee of: Galapagos, A. Kavanaugh Consultant for: Galapagos, M. Genovese Consultant for: Gilead, Galapagos, Abbvie, Lilly, Pfizer

DOI: 10.1136/annrheumdis-2018-eular.1947

\section{SAT0201 REAL WORLD EVIDENCE ON SWITCHING BETWEEN ETANERCEPT AND ITS BIOSIMILAR IN RHEUMATIC DISEASES}

R. Alten ${ }^{1}$, H. Jones ${ }^{2}$, C. Curiale ${ }^{3}$, T. Meng ${ }^{4}$, L. Lucchese ${ }^{5}$, C. Miglio ${ }^{5} .{ }^{1}$ SchlossparkKlinik, University Medicine Berlin, Berlin, Germany, ${ }^{2}$ Pfizer Inc., Collegeville, PA, United States, ${ }^{3}$ Pfizer, Rome, Italy, ${ }^{4}$ Pfizer Pharma GmbH, Berlin, Germany, ${ }^{5}$ IQVIA, London, United Kingdom

Background: Etanercept (Etn) is a biologic agent (BA) that has been proved to be successful in the treatment of rheumatic diseases, by acting as tumour necrosis factor inhibitor, but it is costly. In February 2016, the first etanercept biosimilar (EtnBS) was launched in Germany as a relatively cheaper alternative.

Objectives: In a recent study using the German Longitudinal Prescriptions database IQVIA (LRx) we showed that despite many patients (approximately 50\%) were moved from EtnBA to EtnBS treatment over the year following its launch, some $(10 \%)$ switched back to the original product after few months ${ }^{1}$. As new data are available from the database, the objective of this second analysis was to evaluate switching-back dynamics over longer follow-up durations.

Methods: The German LRx covers prescription data from January 2008, representing approximately $60 \%$ of the German statutory health insurance market. The study period was from February 2016, date of EtnBS launch in Germany, to August 2017 (last available data). Patients receiving first EtnBS prescription (index date) during the study period were retrospectively identified and separated into two groups based on treatment received in the 12 months prior to index date: treatment-naïve patients (no prior biologic treatment) and patients previously treated with EtnBA or other anti-TNF biologics. For the latter group, the cumulative proportion of patients switching back from EtnBS to EtnBA and the median time to the switch-back were evaluated over 3 time periods corresponding to dates of new data availability within the data source: February 2016-September 2016 (1), February 2016-March 2017 (2), and February 2016-August 2017 (3). The results were compared using the chi square test with significance set at $p<0.05$. Data on the market share for biologic agents and their biosimilars in rheumatic diseases are also shown on a monthly basis, between January 2015 and August 2017.

Results: A total of 707, 1,607 and 2,229 patients were identified who received prior EtnBA treatment before switching to EtnBS in time periods 1, 2 and 3 respectively. Of these patients, the proportion of those who switched back to EtnBA significantly $(p<0.05)$ increased over time: $53(7 \%)$ in time period $1,153(10 \%)$ in time period 2 and $320(14 \%)$ in time period 3 . Patients generally switched back to the biologic agent within 3-4 months of starting EtnBS treatment. The use of EtnBS by rheumatologists has constantly grown since February 2016, with a market share of 6\% in Dec 2016 increased to $12 \%$ in August 2017.

Conclusions: This study confirmed previous findings on switching dynamics between EtnBA and its biosimilar. In addition, the study shows that despite a constant increase in the use of EtnBS since its launch, from September 2016 to August 2017, the proportion of patients who switch back to EtnBA after 3-4 months of initiating EtnBS has doubled.

\section{REFERENCE:}

[1] Alten R, et al. ISPOR 20th Annual European Congress in Glasgow, Scotland 2017 November 4-8.

Disclosure of Interest: R. Alten Grant/research support from: The study was sponsored by Pfizer, H. Jones Employee of: Pfizer, C. Curiale Employee of: Pfizer, T. Meng Employee of: Pfizer, L. Lucchese Grant/research support from: The study was sponsored by Pfizer, C. Miglio Grant/research support from: The study was sponsored by Pfizer

DOI: 10.1136/annrheumdis-2018-eular.6076

\section{SAT0202}

COMPARISON OF THE BIOAVAILABILITY OF A SINGLE DOSE OF CERTOLIZUMAB PEGOL INJECTED BY PREFILLED SYRINGE OR BY ELECTRO-MECHANICAL AUTO-INJECTION E-DEVICE: A PHASE 1, OPEN-LABEL, RANDOMISED, PARALLEL GROUP, SINGLE-CENTRE BIOEQUIVALENCE STUDY

R. Oliver ${ }^{1}$, B. VanLunen ${ }^{2}$, I. Mountian ${ }^{3}$, E. Brown ${ }^{2}$, D. Tatla ${ }^{2} .{ }^{1}$ UCB Pharma, Slough, United Kingdom, ${ }^{2}$ UCB Pharma, Raleigh, NC, United States, ${ }^{3}$ UCB Pharma, Brussels, Belgium

Background: When administered subcutaneously (SC) using a pre-filled syringe (PFS), the anti-TNF certolizumab pegol (CZP) has a half-life of $\sim 14$ days and has shown good bioavailability $(\sim 80 \%)$ at all tested doses in healthy volunteers and patients with rheumatoid arthritis. ${ }^{1,2} \mathrm{~A}$ reusable electro-mechanical auto-injection device (e-Device), ava ${ }^{\oplus}$, was recently approved in the EU, ${ }^{1}$ providing an alternative SC-delivered CZP option in addition to the PFS and autoinjector device (AutoClicks $\left.{ }^{\top}\right){ }^{1,3}$

Objectives: To determine if a single $200 \mathrm{mg} \mathrm{CZP}$ dose is bioequivalent when delivered SC by PFS or e-Device, and to assess the safety and tolerability of both administration methods.

Methods: NCT02806219 was a phase 1, open-label, randomized, parallel group, single-center bioequivalence study. Healthy volunteers were randomized $1: 1$ to receive CZP via either the PFS or e-Device. Primary outcomes were assessed using the pharmacokinetic per-protocol set (PK-PPS): maximum CZP plasma concentration $\left(\mathrm{C}_{\max }\right)$, area under the plasma concentration vs time curve $(A \cup C)$, and $A \cup C$ from baseline (BL) to final data point $\left(A \cup C_{(0-t)}\right)$. At $B L$ (Day 1), volunteers received a single $200 \mathrm{mg}$ CZP dose. CZP plasma concentrations were measured on Day 1 prior to CZP administration, at 12 hours (h) post-dose, and on Days $2-7,10,14,21,28,42,56$, and 70 . Safety and tolerability were assessed using the safety set (all receiving a CZP dose) via reported treatment-emergent adverse events (TEAEs), serious AEs, and adverse device events (ADEs: AEs considered by the investigator to be related to/caused by the device). An injection site pain visual analog scale (VAS; $0-100 \mathrm{~mm}$ ) was completed immediately postinjection $(0 \mathrm{~h})$ and $1 \mathrm{~h}$ post-injection.

Results: 100 healthy volunteers were randomized to receive CZP either via PFS $(n=50)$ or e-Device $(n=50)$. The mean plasma CZP concentration vs time profiles for the e-Device and PFS were comparable. Point estimates and $90 \%$ confidence intervals (Cls) for test/reference geometric mean ratios in $\mathrm{C}_{\max }$ and AUC were contained within bioequivalence limits of $80-125 \%$ (table 1). Both administration methods were equally well tolerated; all reported TEAEs were mild or moderate, with no ADEs or injection site reaction TEAEs. Mean VAS pain scores were low at $0 \mathrm{~h}$ (PFS: 10.7 [SD 14.3], e-Device: 18.0 [24.4]) and $1 \mathrm{~h}$ (1.4 [2.9] vs 2.7 [7.0]).

\begin{tabular}{|c|c|c|c|c|c|c|c|}
\hline & \multicolumn{2}{|r|}{ Reference } & & Test & \multicolumn{2}{|c|}{ TestiReference } & \multirow{3}{*}{$\begin{array}{l}\text { ANCOVA } \\
\text { CV (\%) }\end{array}$} \\
\hline & \multicolumn{2}{|c|}{ CZP 200mg PFS } & \multicolumn{2}{|c|}{ CZP 200mg eDevice } & \multirow[b]{2}{*}{$\begin{array}{c}\text { Point } \\
\text { estimate }\end{array}$} & \multirow[b]{2}{*}{$90 \% \mathrm{Cl}$} & \\
\hline & $\mathrm{n}$ & $\begin{array}{l}\text { Geometric } \\
\text { LS Mean } \\
(95 \% \mathrm{Cl})\end{array}$ & $\mathrm{n}$ & $\begin{array}{l}\text { Geometric } \\
\text { LS Mean } \\
(95 \% \mathrm{Cl})\end{array}$ & & & \\
\hline$C_{\max }(\mu g / m L)$ & 48 & $\begin{array}{c}28.8 \\
(27.1,30.5)\end{array}$ & 50 & $\begin{array}{c}28.7 \\
(27.1,30.4)\end{array}$ & 1.00 & $0.93,1.07$ & 20.6 \\
\hline$A \cup C_{(0, n)}(\mu \mathrm{d} \mathrm{d} / \mathrm{mL})$ & 47 & $\begin{array}{c}670.8 \\
(623.7,721.5)\end{array}$ & 50 & $\begin{array}{c}668.7 \\
(623.1,717.7)\end{array}$ & 1.00 & $0.92,1.09$ & 25.6 \\
\hline AUC ( $\mu \mathrm{g} \mathrm{d} / \mathrm{mL})$ & 45 & $\begin{array}{c}701.8 \\
(653.3,754.0)\end{array}$ & 49 & $\begin{array}{c}688.6 \\
(643.0,737.6)\end{array}$ & 0.98 & $0.90,1.07$ & 24.6 \\
\hline
\end{tabular}

PK-PPS. ANCOVA: analysis of covariance; AUC: area under the curve; $A_{U C} C_{(0-1):}$ : AUC from baseline to final data point; $\mathrm{Cl}$ : confidence interval; $\mathrm{C}_{\text {max }}$ : maximum plasma concentration; $\mathrm{CV}$ : coefficient of variation; d: day; CZP: certolizumab pegol; LS: least squares; PFS: pre-filled syringe; PK-PPS: pharmacokinetic perd: day; CZP:
protocol set.

Conclusions: CZP $200 \mathrm{mg}$ doses were bioequivalent whether administered by PFS or e-Device. The SC-delivered CZP injections were well tolerated in healthy volunteers when using either method.

\section{REFERENCES}

[1] EMA. Summary of Product Characteristics (Cimzia) 2017.

[2] Choy EHS, et al. Rheumatology (Oxford) 2002;41:1133-7.

[3] Astruc B, et al. Ann Rheum Dis 2016;75:1003(AB0301).

Acknowledgements: This study was funded by UCB Pharma. We thank the healthy volunteers in addition to the investigators and their teams who contributed to this study. Editorial services were provided by Costello Medical.

Disclosure of Interest: R. Oliver Employee of: UCB Pharma, B. VanLunen Employee of: UCB Pharma, I. Mountian Employee of: UCB Pharma, E. Brown Employee of: UCB Pharma, D. Tatla Employee of: UCB Pharma DOI: 10.1136/annrheumdis-2018-eular.2422 